So, for the same value of $h$, namely, $\bar{h}$, there are two values of $\theta$, namely, $\bar{\theta}$ and $\bar{\theta}^{\prime}$, which is absurd, since $\theta$ is single-valued.

(b) If the discontinuity is of the second kind, then there must be a sequence $\left\{h_{n}\right\}$, tending to $\bar{h}$, for which the corresponding sequence $\left\{\xi_{n}\right\}$ does not tend to any limit. Therefore two values $k_{1}$ and $k_{2}$ of $h$ can always be found as near as we please to $\bar{h}$ such that the corresponding values $\eta_{1}$ and $\eta_{2}$ of $\xi$ differ from each other by a quantity greater than a suitably prescribed positive quantity $\delta$. But, from $(\mathrm{M}), f(h) / h$ and, consequently, $f^{\prime}(\xi)$ are continuous functions of $h$ at $\bar{h}$. Therefore $\xi$ must be multiple-valued at $\vec{h}$, which is absurd, since $\theta$ is singlevalued.

The University of Calcutta

\title{
A NUMERICAL FUNCTION APPLIED TO CYCLOTOMY
}

BY EMMA T. LEHMER

A function $\phi_{2}(n)$ giving the number of pairs of consecutive integers each less than $n$ and prime to $n$, was considered first by Schemmel.* In applying this function to the enumeration of magic squares, D. N. Lehmer $\dagger$ has shown that if one replaces consecutive pairs by pairs of integers having a fixed difference $\lambda$ prime to $n=\prod_{i=1}^{t} p_{i}{ }^{\alpha_{i}}$, then the number of such pairs $(\bmod n)$ whose elements are both prime to $n$ is also given by

$$
\phi_{2}(n)=\prod_{i=1}^{t} p_{i}^{\alpha{ }^{-1}}\left(p_{i}-2\right) \text {. }
$$

As is the case for Euler's totient function $\phi(n)$, the function $\phi_{2}(n)$ obviously enjoys the multiplicative property $\phi_{2}(m) \phi_{2}(n)$ $=\phi_{2}(m n),(m, n)=1, \phi_{2}(1)=1$. In what follows we call an integer simple if it contains no square factor $>1$. For a simple number $n$ we have the following analog of Gauss' theorem:

$$
\sum_{\delta \mid n} \phi_{2}(\delta)=\phi(n)
$$

* Journal für Mathematik, vol. 70 (1869), pp. 191-2.

$\dagger$ Transactions of this Society, vol. 31 (1929), pp. 538-9. 
where $n$ is simple and where the summation extends over all the divisors of $n *^{*}$ Using Dedekind's inversion formula, we can write

$$
\sum_{\delta \mid n} \phi(n / \delta) \mu(\delta)=\phi_{2}(n),
$$

where $n$ is simple and where $\mu(n)$ is Merten's inversion function.*

It is the purpose of this note to develop another property of $\phi_{2}(n)$, true only for simple numbers, and apply it to the evaluation of the discriminants and resultants of cyclotomic equations.

Let $\left\{\lambda_{k}\right\}$ denote the set of numbers less than $n$ and prime to $n$, and let $\lambda$ be any number of this set. Then it follows at once from D. N. Lehmer's result, that there are $\phi_{2}(n)$ numbers prime to $n$ in the set $\left\{\lambda+\lambda_{k}\right\}$. Let us inquire how many numbers in the set $\left\{\lambda+\lambda_{k}\right\}$ have with $n$ a greatest common divisor $\Delta$.

THEOREM 1. If $\Delta$ is a divisor of the simple number $n$, and if $\lambda$ is any fixed number prime to $n$, and if $\lambda_{k}(k=1,2, \cdots, \phi(n))$ runs over a complete set of numbers $<n$ and prime to $n$, then there are $\phi_{2}(n / \Delta)$ multiples of $\Delta$ prime to $n / \Delta$ in the set $\left\{\lambda+\lambda_{k}\right\}$.

Proof. Let $n=\Delta \prod_{i=1}^{h} p_{i}$. Instead of the set $\left\{\lambda_{k}\right\}$ consider the set

$$
1,2,3, \cdots, n \text {. }
$$

The set

$$
\lambda+1, \lambda+2, \lambda+3, \cdots, \lambda+n,
$$

taken modulo $n$, is the set (I) in some order. In the set (II) there are $n / \Delta$ multiples of $\Delta, \phi(n / \Delta)$ of which are prime to $n / \Delta$. But this result applies to the set (II) instead of the desired set $\left\{\lambda+\lambda_{k}\right\}$. We must therefore exclude those multiples of $\Delta$ from the set (II) which have arisen from the addition of $\lambda$ to those numbers of the set (I) which do not belong to the set $\left\{\lambda_{k}\right\}$. It is clear that none of the $\phi(n / \Delta)$ multiples of $\Delta$ mentioned above were obtained by adding $\lambda$ to numbers not prime to $\Delta$. We have then to exclude only multiples of $\Delta$ obtained by adding $\lambda$ to numbers not prime to $\prod_{i=1}^{h} p_{i}$.

In (I) there are $n / p_{\nu}$ multiples of $p_{\nu}$. Adding $\lambda$ to each of these, we obtain a subset $\left(\mathrm{II}_{\nu}\right)$ of (II),

* Dickson, History of the Theory of Numbers, Chap. 19. 


$$
p_{\nu}+\lambda, 2 p_{\nu}+\lambda, 3 p_{\nu}+\lambda, \cdots, \frac{n}{p_{\nu}} p_{\nu}+\lambda .
$$

Taken modulo $n / p_{\nu}$, the set $\left(\mathrm{II}_{\nu}\right)$ is a complete set of incongruent residues. Hence in this set there are $\left(n / p_{\nu}\right) \Delta$ multiples of $\Delta$, not all of which, however, are prime to $n / \Delta$. In fact in the set $\left(\mathrm{II}_{\nu}\right)$ there are $n /\left(p_{\nu} \Delta p_{1}\right)$ multiples of $p_{1}, n /\left(p_{\nu} \Delta p_{2}\right)$ multiples of $p_{2}$ etc. to be excluded. But this excludes twice the multiples of $p_{1} p_{2}, p_{1} p_{3}, \cdots$, etc. These have to be restored once. By the well known principle of cross-classification, we find that the number of multiples of $\Delta$ prime to $n / \Delta$ in the set $\left(\mathrm{II}_{v}\right)$ is

$$
\frac{n}{p_{\nu} \Delta}-\sum \frac{n}{p_{\nu} \Delta p_{i}}+\sum \frac{n}{p_{\nu} \Delta p_{i} p_{j}}-\cdots=\phi\left(\frac{n}{p_{\nu} \Delta}\right) .
$$

As $\nu$ runs from 1 to $h$, we get subsets $\left(I_{1}\right),\left(I_{2}\right), \cdots,\left(I_{h}\right)$ of the set (II). The total amount we must subtract, at this stage, from $\phi(n / \Delta)$ to allow for numbers in the set (I) not prime to $n$ is

$$
\sum_{\nu=1}^{h} \phi\left(\frac{n}{p_{\nu} \Delta}\right)
$$

But again this excludes twice those multiples of $\Delta$ in the set (II) which correspond to multiples of $p_{1} p_{2}, p_{1} p_{3}, \cdots$, in the set (I). These multiples must be restored once and, using once more the principle of cross-classification, we find that the number of multiples of $\Delta$ prime to $n / \Delta$ in the set $\left\{\lambda+\lambda_{k}\right\}$ is given by

$$
\begin{aligned}
\phi\left(\frac{n}{\Delta}\right)-\sum \phi\left(\frac{n}{p_{i} \Delta}\right)+\sum \phi\left(\frac{n}{p_{i} p_{j} \Delta}\right)-\cdots \\
=\sum_{\delta \mid n / \Delta} \phi\left(\frac{n}{\Delta \delta}\right) \mu(\delta)=\phi_{2}\left(\frac{n}{\Delta}\right),
\end{aligned}
$$

by (2). Hence the theorem is proved.

The preceding theorem is not true for non-simple numbers. The corresponding theorem for Euler's $\phi(n)$ states that the number of numbers $m<n$ such that $(m, n)=\Delta$, is $\phi(n / \Delta)$. This is true for all $n$. The simple proof of this theorem cannot be extended to Theorem 1 .

It is important to notice that the result of Theorem 1 is independent of the choice of $\lambda$. As $\lambda$ runs over all the numbers 
in the set $\left\{\lambda_{k}\right\}$ one obtains a matrix $\left\|a_{i j}\right\|=\left\|\lambda_{i}+\lambda_{j}\right\|$ in which by Theorem 1 there are $\phi(n) \phi_{2}(n / \Delta)$ multiples of $\Delta$ prime to $n / \Delta$.

The above result furnishes a ready method of obtaining the explicit formula for the discriminant* of the cyclotomic equation $Q_{n}(x)=0$, whose roots are the primitive $n$th roots of unity without repetition, for $n$ a simple number. The following lemma will enable us to obtain the discriminant for a general $n$.

Lemma. If $f(x)=g\left(x^{m}\right)$ is a polynomial of degree $k$ in $x^{m}$, and if the discriminant of $g(x)$ is $D_{g}$, then the discriminant of $f(x)$ is

$$
D_{f}=a_{k}{ }^{2} \cdot m^{m k} D_{g}^{m},
$$

where $a_{k}$ is the constant term of $g(x)$.

Proof. Let $\rho_{i}(i=1,2, \cdots, k)$ be the roots of $g(x)=0$, and let one of the values of $\rho_{i}{ }^{1 / m}$ be $\theta_{i}$. Then all the roots of $f(x)=0$ are given by $\theta_{i} \epsilon^{\tau}$, where $\epsilon$ is a primitive $m$ th root of unity and $\tau=1,2, \cdots, m$. Then the discriminant of $f(x)$ can be written

$$
D_{f}=\prod_{i<j \leqq k} \prod_{\tau, \nu=1}^{m}\left(\theta_{i} \epsilon^{\nu}-\theta_{j} \epsilon^{\tau}\right)^{2} \cdot \prod_{i=1}^{k} \prod_{\tau<\nu \leqq m}\left(\theta_{i} \epsilon^{\nu}-\theta_{i} \epsilon^{\tau}\right)^{2} .
$$

The first product is

$$
\begin{aligned}
\prod_{i<j \leqq k} \prod_{\nu=1}^{m}\left[\left(\theta_{i} \epsilon^{\nu}\right)^{m}-\theta_{j}^{m}\right]^{2} & =\prod_{i<j \leqq k}\left(\theta_{i}^{m}-\theta_{j}^{m}\right)^{2 m} \\
& =\prod_{i<j \leqq k}\left(\rho_{i}-\rho_{j}\right)^{2 m}=D_{g}^{m} .
\end{aligned}
$$

For the second product we have

The product

$$
\begin{aligned}
\prod_{i=1}^{k} \theta_{i}{ }^{2 m} \prod_{\tau<\nu \leqq m}\left(\epsilon^{\nu}-\epsilon^{\tau}\right)^{2} & =\prod_{i=1}^{k} \rho_{i}{ }^{2} \prod_{\tau<\nu \leqq m}\left(\epsilon^{\nu}-\epsilon^{\tau}\right)^{2} \\
& =a_{k}{ }^{2} \prod_{\tau<\nu \leqq m}\left(\epsilon^{\nu}-\epsilon^{\tau}\right)^{2 k} .
\end{aligned}
$$

$$
\prod_{\tau<\nu \leqq m}\left(\epsilon^{\nu}-\epsilon^{\tau}\right)^{2}
$$

* Rados, Journal für Mathematik, vol. 131 (1906), pp. 49-55, has calculated the discriminant using the derivative definition. The reduction to a simple number would have simplified his proof considerably. 
is the discriminant of $x^{m}-1$, which is known to be $m^{m}$. Hence the lemma follows at once.

We shall proceed to find the discriminant of $Q_{n}$ for $n$ a simple number.

THEOREM 2. If $n$ is simple, the discriminant of $Q_{n}(x)=0$ is given by

$$
D_{n}=(-1)^{\phi(n) / 2} \prod_{i=1}^{t} p_{i}^{\phi\left(n / p_{i}\right) \phi_{2}\left(p_{i}\right)} .
$$

Proof. Since the roots of $Q_{n}(x)=0$ are $e^{2 \pi i \lambda_{k} / n}$ we have

$$
\begin{aligned}
D_{n} & =\prod_{\lambda_{k}<\lambda_{m}}\left(e^{2 \pi i \lambda_{k} / n}-e^{2 \pi i \lambda_{m} / n}\right)^{2} \\
& =(-1)^{\phi(n) / 2} \prod_{\lambda_{k} \neq \lambda_{m}}\left(e^{2 \pi i \lambda_{k} / n}-e^{2 \pi i \lambda_{m} / n}\right) \\
& =\left[\prod e^{2 \pi i \lambda_{k} / n}\right]_{\phi(n)-1} \prod_{\lambda_{k} \neq \lambda_{m}}\left(1-e^{2 \pi i \lambda_{m} / n}\right) .
\end{aligned}
$$

The first product is unity, since $Q_{n}(0)=1$. To evaluate the second product we observe that there is a one to one correspondence between the numbers $\left\{\lambda_{m}-\lambda_{k}\right\}(\bmod n)$ and the elements of the matrix discussed above. The condition $\lambda_{m} \neq \lambda_{k}$ excludes from the matrix all the multiples of $n$. For a divisor $\Delta$ of $n$, the factors of the second product may be grouped into sets of $\phi(n / \Delta)$ elements each, in which $\lambda_{m}-\lambda_{k}$ runs over all the numbers prime to $n / \Delta$. By Theorem 1 there are $\phi(n) \phi_{2}(n / \Delta) / \phi(n / \Delta)=\phi(\Delta) \phi_{2}(n / \Delta)$ such sets for each $\Delta<n$. Hence

$$
D_{n}=(-1)^{\phi(n) / 2} \prod_{\Delta \neq n}\left[Q_{n / \Delta}(1)\right]^{\phi(\Delta) \phi_{2}(n / \Delta)} .
$$

It is known* that $Q_{n}(1)$ is $p$ or 1 according as $m$ is a power of a prime $p$, or not. Accordingly we have

$$
D_{n}=(-1)^{\phi(n) / 2} \prod_{i=1}^{t} p_{i}^{\phi\left(n / p_{i}\right) \phi_{2}\left(p_{i}\right)},
$$

which is the theorem.

* N. Trudi, Atti Accademia Napoli, vol. 3 (1866-8), pp. 20-29; Netto, Vorlesungen über Algebra, vol. 1, p. 357. 
To get the discriminant for a general $n$ we make use of the relation*

$$
Q_{n}(x)=Q_{n_{0}}\left(x^{m}\right),
$$

where $n=n_{0} m$, and $n_{0}$ is the largest simple factor of $n$. Hence applying our lemma with $k=\phi\left(n_{0}\right)$ and $a_{k}=1$, we have

$$
D_{n}=D_{n_{0}}^{m} \cdot m^{m \phi\left(n_{0}\right)}=(-1)^{m \phi\left(n_{0}\right) / 2} m^{m \phi\left(n_{0}\right)} \coprod_{i=1}^{t} P_{i}^{m \phi\left(n_{0} / p_{i}\right) \phi_{2}\left(p_{\boldsymbol{i}}\right)} .
$$

But, since $\phi\left(n_{0} / p_{i}\right) \phi_{2}\left(p_{i}\right)=\phi\left(n_{0}\right)-\phi\left(n_{0}\right) /\left(p_{i}-1\right)$ and $m \phi\left(n_{0}\right)$ $=\phi(n)$, the discriminant can be written in the following general form:

$$
D_{n}=(-1)^{\phi(n)} \frac{n^{\phi(n)}}{\prod_{i=1}^{t} P_{i}^{\phi(n) /\left(p_{i}-1\right)}} .
$$

We shall next consider the resultant $R_{m, n}$ of any $Q_{m}$ and $Q_{n}$. First let $m$ and $n$ be simple numbers $(m \neq n)$. Then we have

Theorem 3. If $m, n$ are simple numbers, †and $m<n$, then

where $p$ is a prime.

$$
R_{m, n}= \begin{cases}p^{\phi(m)}, & \text { if } n / m=p, \\ 1, & \text { if } n / m \neq p,\end{cases}
$$

Proof. Let $(m, n)=d$, and $m=m_{1} d, n=n_{1} d$. Then if $\lambda$ and $\lambda^{\prime}$ run over numbers prime to $m$ and $n$, respectively, we have

$$
\begin{aligned}
R_{m, n}=\prod\left(e^{2 \pi i \lambda / m}-e^{2 \pi i \lambda^{\prime} / n}\right) \\
=\left[\prod e^{2 \pi i \lambda / n}\right]^{\phi(n)} \cdot \Pi\left(1-e^{2 \pi i\left(\lambda^{\prime} m-\lambda n\right) /(m n)}\right) .
\end{aligned}
$$

The product $\left[\Pi e^{2 \pi i \lambda / m}\right]^{\phi(n)}$ is equal to 1 . The fractions occurring in the last product may be written $\left(\lambda^{\prime} m_{1}-\lambda n_{1}\right) /\left(m_{1} n_{1} d\right)$. The numerators are all prime to $m_{1} n_{1}$ but may not be prime to $d$. Modulo $d$, the numbers $\lambda^{\prime} m$ and $\lambda n$ run, respectively, $\phi\left(m_{1}\right)$ and $\phi\left(n_{1}\right)$ times over the complete set of numbers prime to $d$. If $\delta$ is any divisor of $d$, then by Theorem 1 , there are loc. cit.

* Trudi, Annali di Matematica, (2), vol. 2 (1868-9), pp. 160-2; Netto,

$\dagger R_{1,2}=-R_{21}=2$. For all other values of $m, n, R_{m, n}=R_{n, m}$. 
$\phi\left(m_{1}\right) \phi\left(n_{1}\right) \phi(d) \phi_{2}(d / \delta)$ multiples of $\delta$ prime to $d / \delta$ in the set $\lambda^{\prime} m_{1}-\lambda n_{1}$. Hence

$$
R_{m, n}=\prod_{\delta \mid d}\left[Q_{m_{1} n_{1} d / \delta}(1)\right]^{\phi(\delta) \phi_{2}(d / \delta)} .
$$

But, by Trudi's theorem quoted above concerning $Q(1)$, the factors of the above product are equal to unity for $\delta<d$. For $d m_{1} n_{1} / \delta$ cannot be a prime. Hence

$$
R_{m, n}=Q_{m_{1} n_{1}}^{\phi(d)}(1)
$$

Again, by Trudi's theorem, this expression is unity except when $m_{1} n_{1}$ is a prime $p$, that is, when $n / m=p$. In this case $R_{m, n}=p^{\phi(m)}$. Hence the theorem.

To find the resultant $R_{m, n}$, where $m$ and $n$ are any distinct integers, we may proceed as follows. Let $m=m_{0} \mu$ and $n=n_{0} \nu$ where $m_{0}$ and $n_{0}$ are the largest simple divisors of $m$ and $n$. Let also $(\mu, \nu)=d,\left(m_{0}, \nu / d\right)=d_{1}$ and $\left(n_{0}, \mu / d\right)=d_{2}$. Finally let $r_{k}$ and $s_{l}$ be the roots of $Q_{m_{0}}(x)=0$ and $Q_{n_{0}}(x)=0$; let $\rho_{k}$ be one of the values of $r_{k}^{1 / \mu}$ and $\sigma_{l}$ be one of the values of $s_{l}^{1 / \nu}$. Then, by (3), all the roots of $Q_{m}(x)=0$ are given by $\rho_{k} \epsilon_{\mu}^{i}$, where $\epsilon_{\mu}$ is a primitive $\mu$ th root of unity, $k=1,2, \cdots, \phi\left(m_{0}\right)$, and $i=1,2, \cdots, \mu$. Similarly the roots of $Q_{n}(x)=0$ are given by $\sigma_{l} \epsilon_{\nu}^{j}$, where $\epsilon_{\nu}$ is a primitive $\nu$ th root of unity, $l=1,2, \cdots, \phi\left(n_{0}\right)$, and $j=1,2, \cdots, \nu$. Hence we may write

$$
\begin{aligned}
R_{m, n} & =\prod_{k=1}^{\phi\left(m_{0}\right)} \prod_{l=1}^{\phi\left(n_{0}\right)} \prod_{i=1}^{\mu} \prod_{j=1}^{\nu}\left(\rho_{k} \epsilon_{\mu}^{i}-\sigma_{l} \epsilon_{\nu}^{j}\right) \\
& =\prod_{k, l} \prod_{i=1}^{\mu}\left(\begin{array}{c}
\nu \rho_{k} \epsilon_{\mu} \\
\epsilon_{\mu}
\end{array}-\sigma_{l}^{\nu}\right)=\prod_{k, l} \prod_{i=1}^{\mu / d}\left(\rho_{k i}^{\nu i d} \epsilon_{\mu}-s_{l}\right)^{d} \\
& =\prod_{k, l}\left(r^{\nu / d}-s^{\mu / d}\right)^{d}=\prod_{k=1}^{\phi\left(m_{0} / d_{1}\right)} \prod_{l=1}^{\phi\left(n_{0} / d_{2}\right)}\left(\bar{r}_{k}-\bar{s}_{l}\right)^{d \phi\left(d_{1}\right) \phi\left(d_{2}\right)},
\end{aligned}
$$

where $\bar{r}$ and $\bar{s}$ are respectively the roots of $Q_{m_{0} / d_{1}}(x)=0$ and $Q_{n_{0} / d_{2}}(x)=0$. Hence

$$
R_{m, n}=R_{m_{0} / d_{1}, n_{0} / d_{2}}^{d \phi\left(d_{1}\right) \phi\left(d_{2}\right)}
$$

Since $m_{0} / d_{1}$ and $n_{0} / d_{2}$ are simple numbers we may apply Theorem 3 and obtain the following result. 
THEOREM 4. Let $m=m_{0} \mu$ and $n=n_{0} \nu$ be any positive integers, where $m_{0}$ and $n_{0}$ are the largest simple divisors of $m$ and $n$. Let $(\mu, \nu)=d,\left(m_{0}, \nu / d\right)=d_{1},\left(n_{0}, \mu / d\right)=d_{2}, a n d^{*} m_{0} d_{2}<n_{0} d_{1}$. Then

Since

$$
R_{m, n}= \begin{cases}p^{d \phi\left(m_{0}\right) \phi\left(d_{2}\right)}, & \text { if } n_{0} d_{1} /\left(m_{0} d_{2}\right)=p, \\ 1, & \text { if } n_{0} d_{1} /\left(m_{0} d_{2}\right) \neq p .\end{cases}
$$

$$
\prod_{\delta / n} Q_{\delta}(x)=x^{n}-1
$$

and since the discriminant of a product of several polynomials is equal to the product of their discriminants times the product of the squares of the resultants of the polynomials taken two at a time, we have the following identity for $n$ a simple number:

$$
n^{n}=\prod_{\delta / n}\left[\left(\frac{n}{\delta}\right)^{2 \phi(\delta)} \prod_{p_{i} / \delta} p_{i}^{\phi\left(\delta / p_{i}\right) \phi_{2}\left(p_{i}\right)}\right] .
$$

If $n$ is not a simple number, the conditions of Theorem 4 are so complicated that the corresponding identity cannot be easily expressed. The following table which gives the discriminants and the resultants belonging to the divisors of 72 illustrates

\begin{tabular}{|c|c|c|c|c|c|c|c|c|c|c|c|c|}
\hline$\delta \quad 1$ & 2 & 3 & 4 & 6 & 8 & 9 & 12 & 18 & 24 & 36 & 72 & $D_{\delta}$ \\
\hline 1 & 2 & 3 & 2 & 1 & 2 & 3 & 1 & 1 & 1 & 1 & 1 & 1 \\
\hline 2 & & 1 & 2 & 3 & 2 & 1. & 1 & 3 & 1 & 1 & 1 & 1 \\
\hline 3 & & & 1 & $2^{2}$ & 1 & $3^{2}$ & $2^{2}$ & 1 & $2^{2}$ & 1 & 1 & 3 \\
\hline 4 & & & & 1 & $2^{2}$ & 1 & $3^{2}$ & 1 & 1 & $3^{2}$ & 1 & $2^{2}$ \\
\hline 6 & & & & & 1 & 1 & $2^{2}$ & $3^{2}$ & $2^{2}$ & 1 & 1 & 3 \\
\hline 8 & & & & & & 1 & 1 & 1 & $3^{4}$ & 1 & $3^{4}$ & $2^{8}$ \\
\hline 9 & & & & & & & 1 & $2^{6}$ & 1 & $2^{6}$ & $2^{6}$ & $3^{9}$ \\
\hline 12 & & & & & & & & 1 & $2^{4}$ & $3^{4}$ & 1 & $2^{4} 3^{2}$ \\
\hline 18 & & & & & & & & & 1 & $2^{6}$ & $2^{6}$ & $3^{9}$ \\
\hline 24 & & & & & & & & & & 1 & $3^{8}$ & $2^{16} 3^{4}$ \\
\hline 36 & & & & & & & & & & & $2^{12}$ & $2^{12} 3^{18}$ \\
\hline 72 & & & & & & & & & & & & $2^{48} 3$ \\
\hline
\end{tabular}
this identity, and gives some idea of the magnitudes under discussion.

$R_{m, n}$ is found in the row marked $m$ and the column marked $n$. The product of the discriminants times the squares of the resultants listed above is found to be $2^{216} 3^{144}=72^{72}$.

Brown University

* This inequality is imposed merely for definiteness and may be reversed at will by interchanging $m$ and $n$. See the previous footnote. 